Vol. 5, No. 3, July 2020,

Webpage: http://rheumres.org

Email: editor@rheumres.org

ISSN:2476-5856

doi: $10.22631 / \mathrm{rr} .2020 .69997 .1096$

(C2020, Iranian Rheumatology Association

\title{
Weekly versus daily leflunomide
}

\author{
Seyed Ruhollah Mousavinasab ${ }^{1}$ (D), Mohammad BagherOwlia ${ }^{2 *}$ (i) \\ ${ }^{I}$ Department of Clinical Pharmacy, School of Pharmacy, Shiraz University of Medical Sciences, Shiraz, Iran. \\ ${ }^{2}$ Department of Internal Medicine, Shahid Sadoughi University of Medical Sciences, Yazd, Iran.
}

Among the most common chronic conditions needing chronic drug therapy are chronic rheumatic diseases, diabetes mellitus, hypertension, depression, psychosis, inflammatory bowel diseases, multiple sclerosis, coronary vascular disease, dyslipidemia and also chronic rheumatic disorders are among. Most researchers and pharmaceutical companies are constantly trying to develop new drugs with more specific measures based on the pathophysiology of the disease in question. Finding an ideal approach in practice guidelines with minimum adverse drug effects is optimum. Post-drug effects deal with the biological effects of a medicine lasting longer than expected from its pharmacological half-life. This could be more evident in many drugs, including hydroxychloroquine, methotrexate, leflunomide, dexamethasone, fluoxetine, and other lipid lowering drugs. This effect not only maintains the therapeutic effect of the drugs, but also reduces the side effects. Leflunomide is an immunomodulatory antirheumatic and disease-modifying drug that is used to treat rheumatoid arthritis as well as other inflammatory arthritis (namely psoriatic arthritis) by inhibiting the synthesis of pyrimidine. Due to the metabolite of leflunomide in the body and the long half-life of this drug, it is possible to prescribe this drug on a weekly basis. Study results have indicated that weekly administration of leflunomide has advantages over daily administration, such as the same therapeutic effect, ease of administration, lower cost of treatment, fewer side effects of medication, and more patient compliance. Thus, based on the results, it can be recommended that leflunomide be administered weekly.

Keywords: Chronic rheumatic disorders, Leflunomide, Weekly administration,

\section{Introduction}

Chronic conditions are a major global concern. Chronic rheumatic diseases, diabetes mellitus, hypertension, depression, psychosis, inflammatory bowel diseases, multiple sclerosis, coronary vascular disease, dyslipidemia and also chronic rheumatic disorders are among the most common chronic conditions requiring chronic drug therapy. Looking for an optimum strategy in managing patients with chronic disorders is very important. Patient convenience, drug safety, and maximum efficacy form the rationale for finding the best treatment in caring for patients with chronic disorders. Throughout the history of rheumatology, great achievements have been made in improving the management of chronic rheumatic disorders using more specific drugs, especially targeted agents and signaling inhibitors, to overcome disabling diseases more precisely. Apart from non-pharmacological management of rheumatic disorders, drug therapy is the cornerstone for treatment in most instances. Therefore, the safety profile of management is crucial. Strategies for reducing adverse drug reactions and maximizing the benefits and compliance with drug therapy should be our major concerns. Most researchers and pharmaceutical companies endeavor to develop new drugs with more specific measures based on the pathophysiology of the disease. However, clinical rheumatologists should consistently search for the optimum management strategy with the available medications. Ongoing modifications to ideas and understanding previous pitfalls make medicine an everchanging science and practice. However, considering the over-emphasis on new medicine and occasional gap between clinical practice and pharmacy disciplines to improve treatment guidelines may impose some burdens for patients in cases of classic medications. For a long period of time, systemic corticosteroids were the standard

Personal non-commercial use only. Rheumatology Research Journal. Copyright (C 2020. All rights reserved

*Corresponding Author: Seyed Ruhollah Mousavinasab, Department of Clinical Pharmacy, School of Pharmacy, Shiraz University of Medical Sciences, Shiraz, Iran. PO-Box: 3538224000, E-mail: srmn48@gmail.com , Telefax: +983538224000 .

And Mohammad BagherOwlia, Shahid Sadoughi Hospital, Shahid Sadoughi University of Medical Sciences, Yazd, Iran. PO-Box: 353 8224100, E-mail: bagherowlia@gmail.com, Telefax: +98 3538224000.

Received: 12 February 2020; Accepted: 27 February 2020 
of care for patients with asthma. High-dose glucocorticoid administration was also the base of treatment for patients with rheumatoid arthritis and polymyositis for years until this drug was replaced with drugs with maximum efficacy and minimal side effects.

\section{Where we are now?}

Today, with emerging new disease-modifying antirheumatic drugs, the unwanted side effects of systemic corticosteroids are seen much less often. However, because of critical situations and the overt need for glucocorticoids, side effects like secondary osteoporosis, cataracts, glaucoma, muscle wasting, hypothalamic pituitary axis suppression, secondary infections, brittle diabetes mellitus, avascular necrosis are still frequently seen in daily practice. All these permanent complications are potentially preventable. Disease-modifying anti-rheumatic drugs are discovered and invented to cover this need for safer longterm treatment. In addition, with new advanced biological therapies, the issues of individual side effects as well as cost are also barriers to the widespread, worldwide use of these drugs.

\section{Where should we go?}

Finding an ideal approach in practice guidelines with minimum adverse drug effects is optimum. From an optimistic point of view, we should never have permanent iatrogenic complications like marrow suppression, avascular necrosis, secondary osteoporosis, organ damage, or any other irreversible systemic complications. To attain this goal, permanent and close collaboration between clinicians and pharmacologists is warranted.

In cases of increased interval of drug administration to minimize side effects, previous studies on aminoglycosides $(1,2)$ have shown that administering them with intervals far from their half-lives makes sense and adverse renal effects are considerably low. Recent articles have reported that antihyperlipidemic agents such as statins (3) may have comparable efficacy with minimal side effects used alternately instead of on a daily basis. This report seems to be more realistic if you deal with a chronic disorder (RA) with drugs known to have a long half-life (HCQ or leflunomide).

\section{Leflunomide}

Leflunomide is an immunomodulatory diseasemodifying antirheumatic drug that is used to treat rheumatoid arthritis and other inflammatory arthritis diseases (namely psoriatic arthritis) by inhibiting the synthesis of pyrimidine $(4,5)$. It is a prodrug and is converted to the active metabolite A771726 (a brand new drug known as teriflunomide) in the body, which is responsible for the activity of leflunomide. Leflunomide has a long half-life of 15 days on average due to the enterohepatic cycle $(4,6,7)$.

\section{Drugs with potential "Post drug" effects}

Post-drug effects refer to the biological effects of a medicine lasting longer than expected from its pharmacological half-life. This could be more evident for many drugs, especially in long-term use.
Hydroxychloroquine (HCQ), methotrexate, leflunomide, amiodarone, dexamethasone, fluoxetine, lipid-lowering drugs, and any drug that has a half-life of more than 24 hours are potential candidates for alternate or even weekly administration. Seen with various antibiotics, including aminoglycosides, azithromycin, erythromycin, and fluoroquinolones, this effect is known as the post-antibiotic effect.

\section{Supporting evidence}

Li-Min et al. evaluated the efficacy and safety of weekly administration of leflunomide in patients with early rheumatoid arthritis with mild to moderate disease severity. In their study, 244 patients who met the inclusion criteria were randomly divided into treatment (leflunomide 50 $\mathrm{mg} /$ week, LEF50) and control (leflunomide $10 \mathrm{mg} /$ day LEF10) groups. Patients in both groups were treated for 24 weeks. The results of this study showed that weekly administration is easy, low cost, and has good safety. This treatment protocol also increased patient compliance despite maintaining the therapeutic properties of leflunomide (6).

Jakez-Ocampo et al. assessed the therapeutic effects of weekly administration of leflunomide at a dose of $100 \mathrm{mg}$ in patients with refractory rheumatoid arthritis. Patients were randomly assigned to two groups receiving either 100 $\mathrm{mg}$ weekly (weekly dose of $100 \mathrm{mg}$ without the loading dose) or conventional therapy (loading dose of $100 \mathrm{mg} /$ day for three days, followed by a daily dose of $20 \mathrm{mg}$ ). Patients were evaluated every other month for the first 6 months and then in the ninth and twelfth months. The results of this study also showed that the administration of leflunomide at a dose of $100 \mathrm{mg}$ per week has therapeutic effects comparable to the traditional dose and, therefore, can be considered as a new treatment strategy (7).

\section{Conclusion}

Considering the long pharmacologic and biologic halflives of leflunomide, this drug could potentially be an "onqueue" drug for weekly use in daily practice as methotrexate.

\section{Conflict of interest}

There are no conflicts of interest with regard to the present study. 


\section{References}

1. Tiwari S, Rehan H, Chandra J, Mathur N, Singh V. Efficacy and safety of a single daily dose of gentamicin in hospitalized Indian children: a quasi-randomized trial. J Antimicrob Chemother 2009; 64(5):1096-01. doi: $10.1093 / \mathrm{jac} / \mathrm{dkp} 330$.

2. Buchholtz K, Larsen CT, Schaadt B, Hassager C, Bruun NE. Once versus twice daily gentamicin dosing for infective endocarditis: a randomized clinical trial. Cardiology 2011; 119(2):65-71. doi: $10.1159 / 000329842$.

3. Marcus FI, Baumgarten AJ, Fritz WL, Nolan Jr PE. Alternate-day dosing with statins. Am J Med 2013; 126(2):99-04. doi: 10.1016/j.amjmed.2012.08.007.

4. Leflunomide. [https://www.uptodate.com].
5. Pfaller B, Pupco A, Leibson T, Aletaha D, Ito S. A critical review of the reproductive safety of Leflunomide. Clin Rheumatol 2020; 39(2):607-12. doi: 10.1007/s10067-019-04819-4.

6. Ren LM, Li R, Chen LN, Zhu P, Gu F, Sun LY. et al. Efficacy and safety of weekly leflunomide for the treatment of early rheumatoid arthritis: a randomized, multi-center study. Int J Rheum Dis 2016; 19(7):65157. doi: 10.1111/1756-185X.12677.

7. Jakez-Ocampo J, Richaud-Patin Y, Simón JA, Llorente L. Weekly dose of leflunomide for the treatment of refractory rheumatoid arthritis: an open pilot comparative study. Joint bone spine 2002; 69(3):30711. doi: 10.1016/s1297-319x(02)00397-4. 